\title{
DISSECTING INDIVIDUAL LIGAND-RECEPTOR BONDS WITH A LAMINAR FLOW CHAMBER
}

\author{
Anne Pierres, Joana Vitte, Anne-Marie Benoliel and Pierre Bongrand \\ Lab. Adhesion and Inflammation, UMR INSERM 600/CNRS 6212 \\ Marseille, France
}

October 29, 2018

This un-edited manuscript was accepted for publication by Biophysical Reviews and
Letters
and published in volume 1, pp 231-257 (2006)

\begin{abstract}
The most important function of proteins may well be to bind to other biomolecules. It has long been felt that kinetic rates of bond formation and dissociation between soluble receptors and ligands might account for most features of the binding process. Only theoretical considerations allowed to predict the behaviour of surface-attached receptors from the properties of soluble forms. During the last decade, experimental progress essentially based on flow chambers, atomic force microscopes or biomembrane force probes allowed direct analysis of biomolecule interaction at the single bond level and gave new insight into previously ignored features such as bond mechanical properties or energy landscapes. The aim of this review is (i) to describe the main advances brought by laminar flow chambers, including information on bond response to forces, multiplicity of binding states, kinetics of bond formation between attached structures, effect of molecular environment on receptor efficiency and behaviour of multivalent attachment, (ii) to compare results obtain by this and other techniques on a few well defined molecular systems, and (iii) to discuss the limitations of the flow chamber method. It is concluded that a new framework may be needed to account for the effective behaviour of biomolecule association.
\end{abstract}

keywords : on-rate, off-rate, bond strength, intermediate state

\section{Introduction : there is a need for a more complete de- scription of molecular interactions}

The main function of proteins may well be to bind to other proteins 11. Also, it is a reasonable guess to assume that nearly half molecular species found on the membranes of living cells are involved in adhesive interactions 2]. Therefore, there is an obvious need for a theoretical framework to predict the outcome of encounters between two free or surface-bound ligand and receptor molecules.

A first quite naive way of fulfilling this task might consist of establishing a compendium of known ligands for each known molecule. Indeed, this primary task may certainly be considered as 
a useful step as exemplified by the success of the well known factsbook series, e.g. 3. However, it has long been understood that such purely qualitative data were insufficient. For many years, the concept of affinity dominated most thinking about complex biological reactions 4 and it might be felt that a good starting point for understanding the binding behaviour of biomolecules might consist of measuring the equilibrium affinity constants of association with their ligands. However, this simple view soon proved insufficient 4. A prominent example is the adhesion of flowing leukocytes to surfaces coated with ICAM-1, a ligand of leukocyte $\beta 2$ integrins, or P-selectin, a ligand of the leukocyte surface mucin PSGL-1. As was first shown by Lawrence and Springer in a well-known paper [5], ICAM-1 can bind to immobile leukocytes and keep them at rest under flow, but it cannot stop rapidly moving leukocytes. Conversely, P-selectin can dramatically slow down moving leukocytes and decrease their velocity by a factor of nearly 100, but it cannot keep them immobile if the hydrodynamic flow is set up after a period of rest. The difference between ICAM-1 and P-selectin could not be ascribed to a different equilibrium constant of association with their ligands, but the authors suggested that this different behaviour could be accounted for by a higher association and dissociation constant of bonds formed by P-selectin, while affinities might be comparable. Another prominent example is the outcome of interaction between $\mathrm{T}$ lymphocytes and antigen presenting cells expressing specific complexes formed between histocompatibility molecules and peptides recognized by $\mathrm{T}$ cell receptors : it is well known that this interaction may result in $\mathrm{T}$ lymphocyte full or partial activation, or even paralysis, depending on the properties of interaction between T-cell receptors and their ligand. It was clearly of utmost interest to relate lymphocyte behaviour to a quantitative interaction parameter. As recently summarized [6], many experiments supported the conclusion that lymphocyte activation was determined by the lifetime of T-cell receptor/ligand interaction rather than equilibrium constant.

Other experiments further challenged the simple view that kinetic constants, i.e. the association on-rate and off-rate, might fully account for the association behaviour of cells or molecules. Thus, it was righfully emphasized that a peculiarity of cell adhesion receptors is that the bonds they form are often sujected to forces [7. Further, bond strength cannot be derived from kinetic paramers. Indeed, chemical alteration of the CD34 ligand of leukocyte L-selectin could selectively alter the resistance of L-selectin/CD34 bond to forces without changing the lifetime of unstressed bonds. Other authors 8 reported that mutated $\alpha 4 \beta 1$ integrins bound fibronectin ligand with affinity and kinetic constants comparable to those found on wild-type molecules, but cells expressing altered molecules displayed impaired adhesion strengthening after initial attachment.

Also, it has long been emphasized that bond formation between two molecules is not an all-ornone process, rather, it involves a number of intermediate binding states [10, 11. This multiplicity was recently illuminated with the outstandingly resolutive biomembrane force probe 9], and as a consequence it was well understood that bond rupture was dependent on bond history [12, 13.

A final point is that there is no general way of deriving the rate of bond formation between surface-bound molecules from the association constants measured on their soluble forms. Indeed, the rate of bond formation between bound receptors is clearly dependent on the distance between anchoring points and imposed orientation. This dependence is determined by molecular length and flexibility as well as molecular environment on the membrane[14. As a consequence of aforementioned data, it seemed warranted to suggest[16] that molecular interactions should be described by at least two functions rather than parameters, namely, the frequency of bond formation as a function of distance between anchoring regions and the frequency of bond dissociation as a function of applied force. In view of recent experiments disclosing the multiplicity of binding states, this simple view was insufficient to provide a satisfactory description of experimental data yielded by 
presently available techniques.

Now, it would be a hopeless task to address experimentally aforementiond problems with conventional techniques allowing only to monitor multiple interactions. Indeed, It is very difficult to interpret results on the rupture of attachment between surfaces linked by multiple bonds if the details of force sharing between bonds and possibility of rebinding for detached molecules are not known[15]. Also, it would be difficult to interpret the association kinetics of receptor-bearing surfaces if molecular contacts were not properly synchronized. It is therefore understandable that the development of methods allowing to study individual ligand-receptor bonds gave a spectacular impetus to the dissection of molecular bonds.

Since the experimental study of ligand-receptor interaction heavily relies on the development of efficient ways of probing single bond formation and dissociation, the aim of the present review is twofold : first, we shall describe specific information obtained on ligand-receptor interaction with a flow chamber method. When it is felt useful, we shall compare these data to other pieces of information obtained with atomic force microscopy or biomembrane force probes. Second, we shall discuss the present limitations of the flow chamber method and pending issues concerning data interpretation.

\section{Experimental dissection of single ligand-receptor bonds with a laminar flow chamber}

\subsection{Basic principles.}

A schematic view of the laminar flow chamber is shown below.

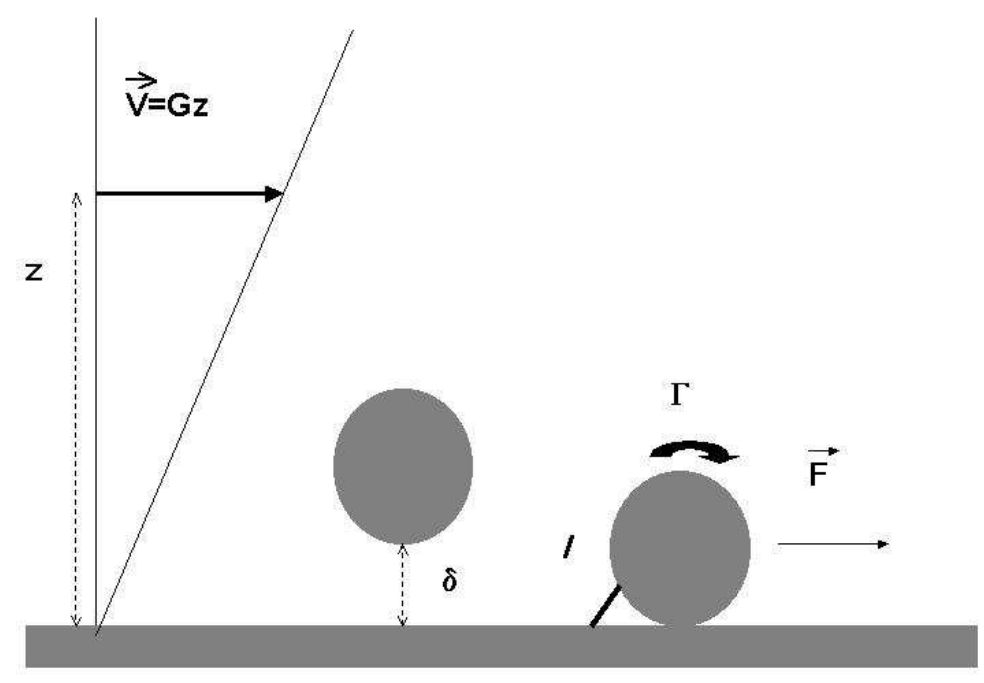

Figure 1: A schematic view of the laminar flow chamber 
A receptor-bearing cell or particle is driven along a ligand-derivatized surface in a laminar shear flow. Many investigators chose to operate under a wall shear rate $\mathrm{G}$ on the order of $100 \mathrm{~s}^{-1}$ or more, thus mimicking hydrodynamic conditions encountered in blood vessels [17. While this is certainly a reasonable choice to study the properties of adhesion receptors mediating leukocyte-endothelium interaction, we found it most rewarding to use 10-100 fold lower wall shear rates. The advantage brought by this choice is twofold : first, the hydrodynamic drag is low enough to allow a single molecular bond to maintain a particle at rest during a reasonable amount of time. Secondly, it is easy to monitor freely moving particles. This allows quantitative study of the frequency of bond formation, which is a currently important issue.

\subsubsection{Kinetics}

Data interpretation requires a thorough understanding of the motion of a free cell-like object near a wall in shear flow. The motion of a neutrally buoyant sphere near a plane in laminar shear flow was solved numerically and results were nicely summarized in an often-quoted paper [18. The main conclusions are as follows : when the distance $z$ between the sphere center and the wall is much higher than the sphere radius, the velocity $U$ is about $G z$, where $G$ is the wall shear rate. However, when the sphere comes close to the surface, its velocity is expected to vanish very slowly. As a consequence, measuring the velocity may allow a very accurate estimate of the sphere-to-surface distance. We found it convenient to use the following polynomial approximation of Goldman's data[19] :

$$
U / a G=\exp \left(0.0037644 \ln (\delta / a)^{3}+0.072332 \ln (\delta / a)^{2}+0.54756 \ln (\delta / a)+0.68902\right)
$$

where $\delta$ is the distance between the sphere and the wall and $a$ is the sphere radius. Two comments may be useful.

First, it may be noticed that $U / a G$ is close to $0.7,0.5$ and 0.4 respectively when $\delta / a$ is about $0.1,0.01,0.001$, corresponding to a distance of $250 \mathrm{~nm}, 25 \mathrm{~nm}$ and $2.5 \mathrm{~nm}$ for a cell-size sphere of 5 micrometer diameter. Obviously, the significance of the lowest values may be questioned, since cell surfaces are not smooth at the nanometer level. The relevance of Eq. (1) to actual cells was checked experimentally [20] : Goldman's theory was concluded to apply to cells provided a phenomenological friction coefficient was added (this was estimated at 74 nanonewton.s/m), and the cell-to-surface distance was about 1.5 micrometer. This gap might be partly due to cell surface protrusions, but other experiments supported the importance of short range repulsion by components of the pericellular cell matrix or glycocalyx 21]

Second, the relevance of this theory to microspheres of $2.8 \mathrm{~m}$ diameter and $1.3 \mathrm{~g} / \mathrm{l}$ density was studied exerimentally [19]. Full agreement was obtained provided brownian motion was included. This required to take care of hydrodynamic interaction between spheres and surfaces. This interaction resulted in increasing the effective friction cofficient for displacements parallel to the wall by the following dimensionless factor :

$$
F_{x}=\exp \left(0.00332 \ln (\delta / a)^{3}+0.019251 \ln (\delta / a)^{2}-0.18271 \ln (\delta / a)+0.32747\right)
$$

Thus, due to vertical Brownian motion, the average distance between spheres and the surface was about $120 \mathrm{~nm}$ (in accordance with Boltzmann's law) and hydrodynamic forces reduced the diffusion coefficient parallel to the wall by about $50 \%$. The interest of using these microspheres instead of cells was that i) surface structure could be better controlled, and ii) computer-assisted image analysis allowed accurate determination of position with better than $50 \mathrm{~nm}$ resolution. 
The results presented above strongly suggest that the distance between a flowing cell or microsphere and a ligand-coated surface may be higher than the length of most adhesion molecules. Indeed, common receptors such as integrins are about $20 \mathrm{~nm}$ length, and especially large adhesion molecules such as P-selectin (CD62L) or its ligand PSGL-1 (CD62L) are about $40 \mathrm{~nm}$ long. Bond formation thus requires a transient decrease of particle-to-surface distance. It is therefore important to consider the details of vertical motion to estimate the rate of surface molecule associations. This vertical motion may be determined by the structure of glycocalyx elements or surface rugosity when cells are studied. When microspheres are considered, the hydrodynamic interaction between the sphere and the wall may reduce diffusion perpendicular to the wall by a factor $F_{z}$ close to $a / \delta$ at low sphere-to-surface distance. The following approximation was found convenient [19] :

$$
F_{z}=\exp \left(0.0057685 \ln (\delta / a)^{3}+0.092235 \ln (\delta / a)^{2}-0.52669 \ln (\delta / a)+0.76952\right)
$$

Another point deserving some emphasis is the duration of particle-surface contact. A sphere flowing close to the surface is expected to rotate with angular velocity (in radian/s) $0.54 U / a$. The relative velocity between the particle surface and the wall is thus $0.46 \mathrm{U}$. If the length of adhesion receptors is of order of $20 \mathrm{~nm}$ and particle velocity is of order of $10 \mathrm{~m} / \mathrm{s}$, molecular encounter duration is therefore of order of $4 \mathrm{~ms}$.

\subsubsection{Mechanics.}

As shown on Fig. 2.1, a particle deposited on the chamber floor is subjected to a drag force $\vec{F}$ and torque $\vec{\Gamma}$. When the particle is spherical, these are given by the following equations [18]:

$$
\begin{aligned}
& \vec{F}=32.05 \mu a^{2} G \\
& \vec{\Gamma}=11.86 \mu a^{3} G
\end{aligned}
$$

where $\mu$ is the medium viscosity. As previously reported [10], the tensile force T exerted on the bond is given by :

$$
T \approx(F+\Gamma / a) \sqrt{a / 2 L}
$$

As a rule of thumb, in a medium of $0.001 \mathrm{~Pa}$.s viscosity such as water, the force (in piconewton) exerted on a bond of $20 \mathrm{~nm}$ length maintaining at rest a sphere of 1.4 micrometer radius in presence of a wall shear rate $G s^{-1}$ is about $0.5 \mathrm{G}$. As shown on $\mathrm{Eq} 6$, the dependence of the force on bond length is fairly weak. When particles are cells, the force on the bond may be much lower due to a higher lever arm. This lever arm may be of order of the sphere radius 22] or even severalfold higher due to the formation of long tethers [23]. In this case, the force is close to the value given in Eq. 4]

\subsection{Detection of single bonds - Lifetime determination}

The first experimental observation of single bond rupture was probably performed in Harry Goldsmith's laboratory 24]. Osmotically sphered erythrocytes were coated with minimal amounts of antibodies and subjected to laminar shear flow in a moving tube allowing cells to remain a substantial amount of time in the field of a fixed microscope. Hydrodynamic forces made erythrocytes encounter each other, then form doublets that rotated, and experienced a growing distractive forces until they were ruptured. This allowed the first determination of so called unbinding forces, leading 
to the estimate that the mechanical strength of an antigen-antibody bond was close to $24 \mathrm{pN}$. However, individual encounters could not be monitored for a sufficient period of time to allow systematic monitoring of bond formation and dissociation.

Only a few years later did a laminar flow chamber allow extensive observation of blood leukocytes flowing along a surface coated with endothelial cells 25. When subjected to a hydrodynamic force of a few piconewtons, cells exhibited multiple transient arrests of widely varying duration. These arrests could be ascribed to well defined interactions between endothelial E-selectin and leukocyte ligand since they were inhibited by specific antibodies. In view of previous theoretical[26] and experimental [24, 27] work, it was assumed that a single molecular bond could mediate cell arrest. Analysing the statistics of arrest duration, it was concluded that the dissociation rate of an Eselectin mediated bond was about $0.5 \mathrm{~s}^{-1}$.

Now, there remained to prove that this approach indeed allowed detection of single bonds, which is by no means trivial 28 .

A reasonable test might consist of performing limiting dilution analysis and checking that arrest frequency was proportional to the first power of ligand or receptor surface density. This was indeed performed by several investigators [10, 22, 29]. However, this cannot be considered as a formal proof that single bonds are actually detected. Indeed, if only double bonds were detected and receptor preparation contained a limited fraction of aggregates required for arrest, a linear relationship would be expected to occur between receptor density and arrest frequency. Other arguments might be obtained by measuring bond frequency and controlling surfaces for molecular distribution. Also, if it is accepted that the wall shear rate is low enough to allow single bond detection, the shortest binding events may reasonably be ascribed to single bonds.

Now, if binding events were mediated by single bonds with a dissociation rate $k_{\text {off }}$, the number of cells or particles remaining bound at time $t$ after initial arrest should be given by the simple relationship :

$$
N(t)=N(0) \exp -k_{o f f} t
$$

However, while this simple relationship was indeed found to hold under some experimental situations 22, 30, it was soon emphasized that

i) Detachment curves obtained with different ligand-receptor models were not linear [31, 10,

ii) This nonlinearity could be accounted for by different mechanisms such as formation of additional bonds after arrest 29] or time-dependent strenghtening of newly formed bonds as a consequence of the occurrence of several energy barriers along the reaction path [10].

iii) Conversely, the occurrence of a linear relationship between $\ln N(t)$ and time could not be considered as a formal proof that single bond lifetimes were indeed measured, if time resolution or the range of measured durations were not sufficient 32 .

Typical detachment curves are shown on Fig. 2. A common procedure consisted of determining dissociation rates by considering only the initial linear part of unbinding plots 7 .

As shown on Table 1, when different ligand-receptor couples were studied, measured bond lifetimes spanned at least a 1000 fold range. Clearly, it would be of high interest to relate experimental bond lifetimes to functional properties of adhesive interaction they mediate, such as reversibility.

Another interesting point is that bond lifetime modulation was found to be used by living cells as a mechanism of controlling adhesion. Thus, integrins constitute an important family of adhesion receptors that are subjected to extensive regulation. The involved mechanisms were studied by many authors, whose studies revealed the functional importance of affinity changes due to a passage between different conformational states. It was therefore important to assay the importance of these 


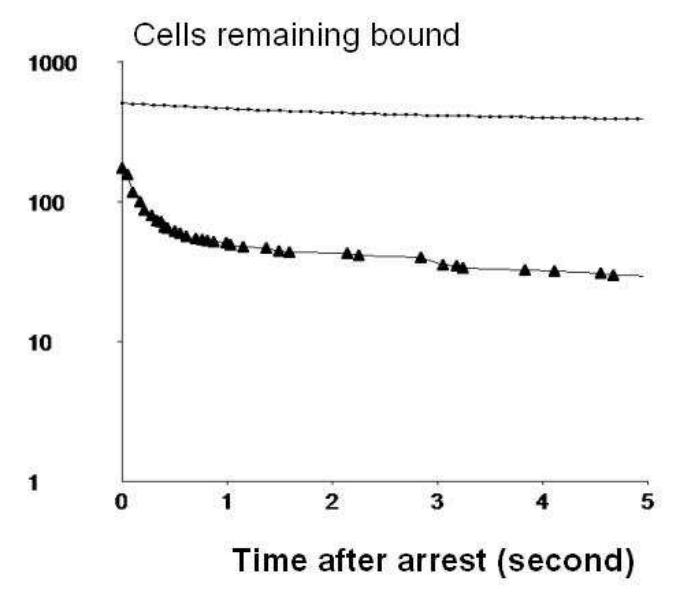

Figure 2: Two typical detachment curves. The detachment plot determined with keratinocytes moving along collagen-coated surfaces (dots) was a straight line, in contrast with the curve determined on monocytic THP-1 cells moving along fibronectin-coated surfaces (triangles)

changes with a flow chamber. Integrin-mediated interaction between collagen surfaces and human keratinocytes was studied : when cells were treated with monoclonal antibodies known to enhance or decrease integrin activity, the bond lifetime was found to vary accordingly : function-enchancing antibodies triggered sixfold increase of bond lifetime, and function inhibiting antibodies triggered up to 13 fold decrease of bond lifetime 30 .

\subsection{Studying the force dependence of bond lifetime}

\subsubsection{Use of the laminar flow chamber to determine force dependence of bond lifetime}

The interest of investigators in the force dependence of bonds was strongly stimulated by a theoretical report from George Bell[26] who elaborated a framework to address interactions between cell surface receptors. He suggested that the dissociation rate $k_{o f f}(F)$ of a bond subjected to a disruptive force $F$ might be calculated with the following equation :

$$
k_{o f f}(F)=k_{o f f}(0) \exp \left(F d / k_{B} T\right)
$$

where $T$ is the absolute temperature, $k_{B}$ is Boltzmann's constant, and $d$ should represent the range of intermolecular forces. Eq. (8) is now referred to as Bell's law. It might be somewhat justified on theoretical grounds, starting from the early Eyring and Kramer theories of reaction rate 33. Now, considering antigen-antibody bonds, Bell estimated $d$ at about $0.5 \mathrm{~nm}$, a value somewhat intermediate between the depth of an antibody cleft and the range of individual interactions. The force coefficient $f_{0}=k_{B} T / d$ might thus be expected to be of order of $8 \mathrm{pN}$. 
The first in-depth experimental check of Bell's idea was performed by Alon and colleagues 22. These authors used a laminar flow chamber to monitor the motion of blood neutrophils driven along surfaces coated with various surfaces densities of $\mathrm{P}$-selectin molecules : the wall shear rate ranged between about 17 and $73 \mathrm{~s}^{-1}$. Chosen values were thus substantially lower that those previously used to mimick blood flow [5. When the surface density of P-selectin was higher than about 30 sites per $\mu m^{2}$, close to the physiological range found on activated endothelial cells, cells interacting with the flow chamber exhibited a jerky motion closely resembling the rolling phenomenon. However, when the surface density of P-selectin was lowered down to 1 site per $\mu m^{2}$, cells moving close to the surface displayed periods of displacement with constant velocity interspersed with transient arrests of varying duration, closely similar to the motion observed with lower wall shear rates and higher ligand densities 25]. These arrests followed first order dissociation kinetics, and the dissociation rate increased when the shear rate was increased. The authors concluded that they analyzed single molecule binding events, and that the dissociation rate followed Bell's law. They were able to derive the force from calculated hydrodynamic forces exerted on cell by a simple geometrical model of cell shape. The zero-force dissociation rate was $0.95 s^{1}$, in close accordance with the estimate of E-selectin bond lifetime 25], and the characteristic distance parameter $d$ of Eq. 8 was $0.049 \mathrm{~nm}$, which was much lower than Bell's estimate. This value was considered to be consistent with those found for hydrogen or calcium coordination bonds.

During the next years, many authors reported estimates of zero-force dissociation rates and force parameters $k_{B} T / d$ for different ligand-receptor couples. Some results are summarized on Table 1. For convenience, rows were ordered according to zero-force dissociation rate.

Table 1 Dissociation parameters measured on different ligand-receptor couples.

\begin{tabular}{lccr}
\hline Ligand-receptor couple & $\begin{array}{c}k_{o f f}(0) \\
s^{-1}\end{array}$ & $\begin{array}{c}k_{B} T / d \\
\text { piconewton }\end{array}$ & Reference \\
\hline CD2/CD48 & 7.8 & 32 & {$[29]$} \\
L-Selectin/CD34 & 6.6 & 218 & {$[35]$} \\
P-Selectin/PSGL1 & 0.95 & 82 & {$[22$} \\
IgG/anti-IgG (transient state) & 0.9 & 53 & {$[10]$} \\
E-Selectin/PSGL1 ? & 0.70 & 138 & {$[35]$} \\
E-SelectinPSGL-1 ? & 0.50 & - & {$[25]$} \\
E-Cadherin/E-Cadherin & 0.45 & 2.4 & {$[36$} \\
Antibody/Group B antigen & 0.04 & 35 & {$[37$} \\
VLA2/Collagen & $0.01-0.2$ & - & {$[30$} \\
IgG/Protein G & 0.006 & 11 & {$[38$} \\
\hline
\end{tabular}

Clearly, zero-force dissociation rates displayed a wide range (a thousandfold) of values, and there was no obvious correlation between $k_{o f f}$ and $k_{B} t / d$.

Now, while Bell's model was considered to account satisfactorily for experimental results found in many studies 39 , additional complexity was soon disclosed.

An important point is the recent experimental demonstration of the existence of so-called catch bonds 40 that had been fancied in the late eighties by Dembo and colleagues 41 . These authors had emphasized that thermodynamics only imposed that the affinity of a given bond be decreased by a disruptive force, but association and dissociation rates (the ratio of which gives the affinity constant) might in principle by either decreased or increased by forces. Thus, they suggested to call slip bonds the molecular bonds that displayed decreased lifetime in presence of disruptive forces, as intuitively expected, and catch bonds the bonds that exhibited opposite behavior. It was only 
recently that Zhu and colleagues were able to convince the scientific community that catch bonds indeed existed, with both a laminar flow chamber and atomic force microscopy. The conclusion was that selectin-mediated bonds might display increased lifetime in presence of a low disruptive force.

\subsubsection{Analysis of bond rupture with other methods : atomic force microscopy and biomembrane force probe}

In a flow chamber, a bond is subjected to a constant force, and measuring bond lifetime makes it intuitively obvious that bond rupture is a stochastic phenomenon that can only be accounted for by a rupture probability.

Now, when molecular attachments were subjected to a rapidly increasing force with an atomic force microscope 42, it appeared intuitively obvious that the relevant parameter was the unbinding force. It seems that the authors felt they were probing the low temperature limit of bond rupture and they were measuring an intrinsic and time-independent parameter. It was only a few years later that the maximum force resisted by a bond subjected to increasing effort was shown to be dependent on the rate of force increase, i.e. the loading rate[43, as expected from Bell's law. Only when the loading rate was varied it became possible to achieve experimental determination of both zero-force dissociation rate and aforementioned parameter $k_{B} T / d$. However, it immediately appeared that single bonds behaved in a less simple way than initially expected, and bond rupture appeared to involve the sequential crossing of several energy barrier. Each crossing appeared to follow Bell's law. Thus, experimental results obtained with different methods cannot be compared before we address this supplementary level of complexity.

\subsection{Analyzing the complexity of ligand-receptor association}

\subsubsection{Dissection of single molecular bonds with a flow chamber}

For many years, it seemed acceptable to view ligand-receptor association as an all-or-none phenomenon : molecules should be either bound or separated, but intermediate complexes were considered as so transient that they should not be detectable unless very rapid kinetic methods were used.

However, when single molecular bonds began being studied, it soon appeared that association indeed involved readily detectable intermediate states. Indeed, when microspheres coated with anti-rabbit immunoglobulin antibodies were made to interact under low shear with surfaces coated with rabbit immunoglobulins, they displayed binding events with a short lifetime of order of 1 second that was fairly insensitive to shear. However, a significant proportion of binding events lasted several seconds or even tens of seconds or more[10. It was concluded that antibody binding was a multiphasic process involving the formation of a transient intermediate state and further strengthening.

Similar conclusions were obtained when streptavidin-coated spheres were made to interact with biotinylated surfaces 11. Transient binding states were observed under conditions where the forces imposed on bonds varied between 3.5 and $11 \mathrm{pN}$. Since this lifetime was much shorter than expected in view of Bell's law and previous experiments done with atomic force microscopy 42, it was concluded that these results revealed the existence of intermediate binding states. Also, since a modest increase of the shear rate and hydrodynamic forces resulted in dramatic decrease of the frequency of binding events without any substantial change of other kinetic parameters, it was suggested that experimental findings might reveal the existence of a still shorter binding state that might be highly 
sensitive to forces. Finally, intermediate binding states with a similar lifetime of order of one second were reported between fibronectin-coated surfaces and monocytic cells expressing integrin receptors for fibronectin 44 .

\subsubsection{Dissecting individual bonds with atomic force microscopy or the biomembrane force probe}

As explained above, when the loading rate was systematically varied, first with the biomembrane force probe 43] and then with atomic force microscopy [45, 46, 47, rate and force constants could be obtained, and it is interesting to compare data obtained on similar molecular systems with different techniques. Selected results obtained on the homotypic interaction between the outer two domains of E-cadherin are shown on Table 2.

Table 2. Rupture of homophilic associations

\begin{tabular}{lccc}
\multicolumn{4}{c}{ between E-cadherin external domains EC1-EC2 } \\
\hline Method & $k_{o f f}(0)$ & $k_{B} T / d$ & Reference \\
& $s^{-1}$ & $p N$ & \\
\hline Flow Chamber & 0.45 & 2.4 & {$[36]$} \\
Biomembrane force probe & & & \\
& $0.5-1$ & $6-7$ & {$[48$} \\
& $8-11$ & $6-7$ & {$[48$} \\
\hline
\end{tabular}

It is concluded that results obtained with the biomembrane force probe and the flow chamber are consistent. The former method displayed additional capacity to reveal a supplementary barrier.

Now, results obtained on the P-selectin model are displayed on Table 3 :

Table 3. Rupture of individual bonds formed between P-selectin and PSGL-1 ligand

\begin{tabular}{lccc}
\hline Method & $\begin{array}{c}k_{o f f}(0) \\
s^{-1}\end{array}$ & $\begin{array}{c}k_{B} T / d \\
p N\end{array}$ & Reference \\
\hline Flow Chamber & 0.95 & 82 & {$[22]$} \\
Atomic Force Microscopy & 0.2 & 30 & {$[46]$} \\
& & & \\
Biomembrane Force Probe & 0.37 & 18 & {$[49$} \\
& $8-12$ & 6 & \\
\hline
\end{tabular}

Here, the flow chamber yielded much higher force constant than the other two techniques. It must be pointed out that the wall shear rate used in this case was much higher than in [36]. Under these conditions, binding events corresponding to the parameters obtained with the other two methods might have been difficult to detect.

Thus, experimental data obtained with the flow chamber, atomic force microscopy and the biomembrane force probe convincingly demonstrated that bond formation and dissociation involve the formation of a hierarchy of binding states of widely varying duration, reflecting the existence of a sequence of barriers in the association or dissociation path, viewed as a valley in the energy landscape of the ligand-receptor molecular complex [50]. Probably the laminar flow chamber is most effective in revealing the properties of the external part of the energy landscape 11, since it allows to probe bonds within a millisecond after formation, subjecting them to forces as low as a few piconewtons. 


\subsection{Experimental study of the rate of bond formation between surface- attached molecules}

\subsubsection{Results obtained with flow chambers}

Determining the rate of bond formation between single molecules is by no means an easy task. A typical experiment would consist of maintaining molecules within binding range for a given amount of time, then determining whether binding occurred. There are three main problems to solve :

First, how can we define two molecules as bound when there is a number of transient binding states, and there is a need to chose an arbitrary threshold duration and/or resistance to force allowing to define two molecules as bound.

Secondly, unless molecules are freely diffusing in a given region of space,the frequency of bond formation is highly dependent on molecular motion, and particularly the motion of binding sites.

Thirdly, if experiments are done with a number of molecules, initial states must be known with higher accuracy. Indeed, if you bring together two surfaces coated with ligand and receptors with low binding probability, it is usually accepted that detected binding events essentially represent single bonds, based on Poisson law. Thus, there is no need to know the initial state of all potential binders. On the contrary, an experimental rate of bond formation is meaningless if the initial state of receptors and ligands is not known.

As a consequence, when laminar flow chambers were first used with low shear rate to monitor bond formation between blood neutrophils and activated endothelial cells 25, the frequency of bond formation was readily determined by monitoring the motion of cells moving in contact with the substrate. However, the experimental frequency $\left(0.04 \mathrm{~s}^{-1}\right)$ could not be used to derive intrinsic molecular parameters since the density and topography of binding molecules were not known.

In a later study[51], a better defined model system was used to study the kinetics of bond formation between surface-attached molecules. Microspheres of 2.8 micrometer diameter were coated with CD48 molecules using antibodies as linkers. They were then driven along surfaces derivatized with CD2, a CD48 ligand, under low shear. Numerous binding events were recorded, and arrest frequency was determined as a function of sphere velocity immediately before arrest. Ignoring brownian motion, it was thus possible to relate the sphere velocity to distance from the surface. This allowed derivation of a tentative relationship between the binding frequency $k(z)$ of two molecules CD2 and CD48 maintained at distance $z$, and the binding rate $P(z)$ of a sphere located ad distance $\mathrm{z}$ from the surface. Denoting the binder surface density on beads and surfaces as $\sigma_{b}$ and $\sigma_{s}$ respectively :

$$
k(z)=\frac{d^{2} P / d z^{2}}{4 \pi^{2} a z \sigma_{b} \sigma_{s}}
$$

The binding frequency was found to be inversely related to the cube of the intermolecular distance, and it was estimated at $0.03 \mathrm{~s}^{-1}$ at $10 \mathrm{~nm}$ separation.

Since the above conclusions relied on unwarranted neglect of Brownian motion, a refined model was used to obtain a more accurate derivation of molecular association rates [52].

Microspheres were coated with recombinant outer domains 1 and 2 (denoted as EC1-2) of cadherin 11, a homotypic adhesion molecule. They were made to interact with molecularly smooth mica surfaces that were coated with EC1-2. In order to ensure proper orientation of bound molecules, adsorption was obtained through hexahistidine moieties that were added to the tail of EC1-2. Multiple trajectories were monitored, and a total of nearly one million positions were recorded. First, acceleration curves were built by plotting the average particle acceleration versus velocity. These 
experimental plots were fitted with curves obtained by computer simulations that accounted for i) Brownian motion parallel and perpendicular to the wall, ii) hydrodynamic interaction between particle and the wall, and iii) van der Waals attraction between spheres and the wall, as accounted for by an adjustable Hamaker constant. Electrostatic interactions were neglected since experiments were performed in media of high (physiological) ionic concentration. Fitting experimental and simulated curves allowed simultaneous determination of both the wall shear rate and Hamaker constant. It may be emphasized that this method was highly sensitive since it allowed to detect forces of order of 10 femtonewtons. Fitted parameters were used to plot the binding frequency of flowing spheres versus dimensionless ratio $U / a G$, where $U$ is the sphere average velocity over a $160 \mathrm{~ms}$ interval, $a$ is the sphere radius, and $G$ is the wall shear rate.

In contrast with standard results from fluid mechanics $18, U / a G$ could only be related to a distribution probability of sphere-to-surface distances. It was thus not feasible to derive analytically the distance-dependent association rate $k_{o n}(d)$ from the binding curve. Thus, in order to proceed, simulated trajectories were built by assuming a simple form for $k_{o n}(d)$ : the rate of association between individual molecules anchored at two points separated by a distance $d$ was taken as a constant $k_{\text {on }}$ when $d$ was lower than a threshold range $R$ and zero elsewhere. Experimental data were consistent with the conclusion that $k_{\text {on }}$ was about $1.210^{-3} \mathrm{~s}^{-1}$ and $R$ was about $10 \mathrm{~nm}$. It may be tempting to compare theses estimates to the 3-dimensional association rates : for this purpose, it may be noticed that the concentration of a molecule restricted to a sphere of $10 \mathrm{~nm}$ radius is about $0.0004 \mathrm{M}$, yielding a tentative three-dimensional $k_{o n}$ of $3 M^{-1} s^{-1}$. This is much lower than expected association rates of soluble molecules. Two possible explanations may be considered :

i) geometric restreints due to rigid coupling between molecules and surfaces may drastically alter binding efficiency.

ii) The concept of association rate should be reconsidered when contact times as low as $1 \mathrm{~ms}$ are considered : indeed, if a minimum contact time is required to allow sufficient bond strenghtening that cadherin-cadherin association be detectable, the probability of bond formation during a cellto-surface approach my not be proportional to contact time. This would make the concept of $k_{\text {on }}$ questionable.

\subsubsection{Comparison with other methods}

Atomic force microscopy was very early used to estimate intermolecular association rates [53, 54. The basic idea [53] was to combine i) estimate of the time required for binding by varying the contact time on a given position, and ii) estimate of the interaction range by combining very slow lateral displacement and continuous binding-unbinding. Studying the interaction between human albumin and polyclonal antibodies, the association rate was estimated at $510^{4} M^{-1} s^{-1}$ which was deemed reasonable. In a later study, the association rate for VE-cadherin was estimated between $10^{3}$ and $10^{4} M^{-1} s^{-1}$. In line with previous remarks, it may be noticed that association rates might appear higher than estimated for the flow chamber since i) the association time was determined in regions

were binding molecules were found. This removed the possibility that inactivity of a fraction of receptors might result in too low values of association rates, and ii) contact times were on the order of $0.1 \mathrm{~s}$, i.e. at least tenfold higher than used in the flow chamber. 


\subsection{Influence of binding site environment of association properties}

\subsubsection{Results obtained with the flow chamber}

While most studies devoted to ligand-receptor interaction are focussed on the relationship between the molecular properties of binding sites and parameters such as association and dissociation rates or mechanical properties, much experimental evidence supports the view that receptor efficiency is higly dependent on a number of structural features that are independent of binding sites, including mode of anchoring, length and flexibility of the connection with cell surfaces, as well as surrounding molecules.

A prominent example is the glycocalyx influence on membrane receptor efficiency. This was demonstrated by elegant experiences related to P-selectin, an endothelial cell receptor interacting with PSGL-1, a mucin ligand expressed by circulating leukocytes [55]. Both P-selectin and PSGL-1 are large molecules of more than $40 \mathrm{~nm}$ length. It was deemed interesting to study the binding capacity of engineered P-selectin with shortened stalk and intact binding sites. Under static conditions, transfected cells expressing wild-type or mutated selectins bound leukocytes with comparable efficiency. However, when experiments were conducted under dynamic conditions in a flow chamber operated at a wall shear rate of about $200 \mathrm{~s}^{-1}$, shortened molecules displayed drastically decreased capacity to tether moving neutrophils. Further, binding capacity under flow was somewhat restored when shortened P-selectin molecules were borne by defective cells with reduced glycocalyx expression.

Similar conclusions were obtained with a flow chamber operated at a much lower shear rate of 10$20 \mathrm{~s}^{-1}$. Phagocyte monolayers were allowed to bind to microbeads interacting with immunoglobulin receptors expressed on cell membranes. Under static conditions, binding was comparable in control cells and cells that had been treated with neuraminidase to reduce their glycocalyx. On the contrary, glycocalyx reduction drastically enhanced microbead uptake under flow [56].

\subsubsection{Other methods aimed at studying individual molecular bonds formed between surface-attached molecules support the importance of binding site environment on bond properties}

The importance of binding site environment was repeadedly revealed in a number of studies. Thus, initial studies made on single bond formation with atomic force microscopy stressed the advantage of depositing a molecular species on soft agar beads 42. Using a dual micropipette system to determine the frequency of bond formation between soft vesicles subjected to transient contact, it was reported that the frequency of bond formation, not dissociation, between ligand and receptor molecules was decreased when molecular length was reduced[57]. Also, the surface forces apparatus was used to demonstrate that the efficiency of association between tethered streptavidin and biotin molecules was increased in proportion with tether length [58].

\subsection{Studying the behaviour of attachements mediated by multiple bonds}

Under most conditions, adhesion is mediated by multiple bonds. Therefore, there is a strong need to relate single and multiple bond properties. It is difficult to achieve this goal by purely theoretical means. Indeed, conclusions are heavily dependent on assumptions that are difficult to assess. Thus, in a recent analysis of the rupture of multiple parallel bond, rupture force was concluded to vary according to the first power, the square root or the logarithm of bond number depending on pa- 
rameters such as force sharing between multiple bonds or possibility of rebinding [15]. Experimental studies are thus needed.

\subsubsection{Quantification of the properties of multivalent attachment with a flow chamber}

There are many examples of cell surface receptors that seem to require a minimal oligomerization for full binding activity. Thus, ICAM-1, the ubiquitous ligand of leukocyte integrin LFA-1, has long been reported to be a dimer on cell membranes. Recently, it was shown that soluble ICAM-1 monomer bound LFA-1 with maximal affinity, but dimerizaton was required to mediate efficient attachment between membrane-bound ICAM-1 and LFA-1[59]. Also, molecular clustering has long been considered as a general mechanism of integrin activation. This was an incentive to quantify the effect of clustering integrin $\alpha 5 \beta 1$ on binding efficiency. The integrin-mediated attachment of monocytic THP-1 cells to fibronectin-coated surfaces was studied with a laminar flow chamber operated at low shear rate[44. Results are summarized on Table 4 :

Table 4. Effect of integrin aggregation on binding efficiency under flow.

\begin{tabular}{lccc}
\hline Cell treatment & Fibronectin concentration $\left(\mathrm{mol} \mathrm{m}^{-2}\right.$ & Binding frequency $\mathrm{mm}^{-1}$ & Initial detachment rate $s$ \\
\hline None & 6,500 & $1.48 \pm 0.07$ & $0.96 \pm 0.10$ \\
anti-integrin mAb & 6,500 & $0.74 \pm 0.07$ & $1.64 \pm 0.26$ \\
Anti-integrin+anti-Ig & 6,500 & $0.93 \pm 0.08$ & $1.18 \pm 0.20$ \\
& & & \\
None & 3,850 & $0.75 \pm 0.08$ & $2.26 \pm 0.40$ \\
anti-integrin mAb & 3,850 & $0.45 \pm 0.04$ & $1.83 \pm 0.29$ \\
anti-integrin+anti-Ig & 3,850 & $1.19 \pm 0.08$ & $1.02 \pm 0.14$ \\
& & & \\
None & 1,400 & $0.21 \pm 0.02$ & $1.94 \pm 0.27$ \\
\hline
\end{tabular}

First, the effect of fibronectin surface density on binding frequency and duration was studied on control cells. When fibronectin surface density was decreased from about 6,500 to 3,850 molecules $/ \mu m^{2}$, both binding frequency and arrest lifetime were decreased, suggesting that attachments might be multivalent. However, when fibronectin surface density was further decreased, binding frequency was strongly decreased without any significant alteration of bond lifetime, which suggested that individual bonds were indeed observed.

Now, when cells were treated with a supposedly neutral anti-integrin antibody, binding frequency on high or intermediated density fibronectin was lowered, and detachment rates were comparable to values ascribed to single bond. The simplest interpretation was that antibodies decreased integrin accessibility, not function.

Finally, when a second (anti-immunoglobulin) antibody was added in order to mediate receptor aggregation (which was checked with confocal microscopy), binding frequency was increased.

A simple interpretation would be that receptor aggregation might favor multivalent initial interactions. This would increase both binding frequency and bond lifetime if it is assumed that binding events detected with a flow chamber might result from the strengthening of shorter - undetectable -binding states. While further work is clearly needed to understand quantitatively the mechanisms of initial adhesion, presented data clearly support the view that cell adhesive behavior may depend on fine properties of the entire energy landscape of ligand-receptor complexes. 


\subsubsection{Studying multivalent attachments with other methods}

The diversity of results described in different reports supports our expectation that there is probably no single rule to predict the behaviour of multivalent attachments. Thus, in an early study of the rupture of streptavidin-biotin bonds with atomic force microscopy 42, the histogram of rupture force frequency displayed a series of peaks appearing as integer multiples of an elementary force quantum (namely $160 \mathrm{pN}$ ) attributed to a single bond, thus suggesting simultaneous breakage of all bonds). In another study made on antigen-antibody interaction[53, the bonds formed by a single divalent antibody appeared as separate events. In a recent report [60, the rupture of multivalent attachments between concanavalin A, a protein bearing four carbohydrate binding sites, and mannose groups suggested nonlinearly additive forces since the rupture forces of attachments involving 1, 2 and 3 bonds respectively were respectively 46,68 and $85 \mathrm{pN}$.

\section{Significance of experimental data and problems raised by flow chamber experiments}

Although flow chambers have now been used for more that ten years to study molecular interactions, some important points still need clarification. We shall now address problems that seem to deserve some attention. Additional information may be found in a recent review 28 .

\subsection{Is there an unambiguous means of proving that single molecular bonds are actually observed ?}

We shall now briefly list some arguments that were sometimes used.

When bond formation is rare, multiple bond formation becomes negligible. This argument was often used with other techniques such as atomic force microscopy. Thus, if only $10 \%$ of approach/retraction cycles are conducive to bond formation, it is often concluded that only $1 \%$ of these cycles will be conducive to multiple bonds. However, this argument is not tenable unless it is assumed that all binding events mediated by single bonds are actually detectable. Otherwise, it may only be concluded that single minimal detectable events are studied.

When binding sites are diluted, the frequency of binding events decreases linearly with respect to binding site density. This argument may not be considered as a fully rigorous proof that single bonds are observed. Indeed, if a proportion of receptors or molecules are aggregated, which is a fairly frequent occurrence with protein samples, it is conceivable that multivalent bond frequency might behave as a linear function of receptor density. Note that this possibility is difficult to rule out since ligand adsorption on a surface may be somewhat irregular even if coating media were carefully deaggregated, e.g. by ultracentrifugation. A sample image of adsorbed fibronectin is shown as an example on Fig 3

A possible way of ruling out this possibility might consist of comparing the frequency of binding events to the expected encounter-frequency of ligand and receptor sites. Clearly, if a high proportion of these encounters resulted in detectable binding, these events should not represent the presence of a rare proportion of aggregates.

Unbinding events follow first order kinetics. As was previously emphasized 32, if the minimal duration of detectable binding events is too high, the rupture of multivalent attachments may seem to follow first order kinetics if the initial nonlinear part of the curve is missed. 


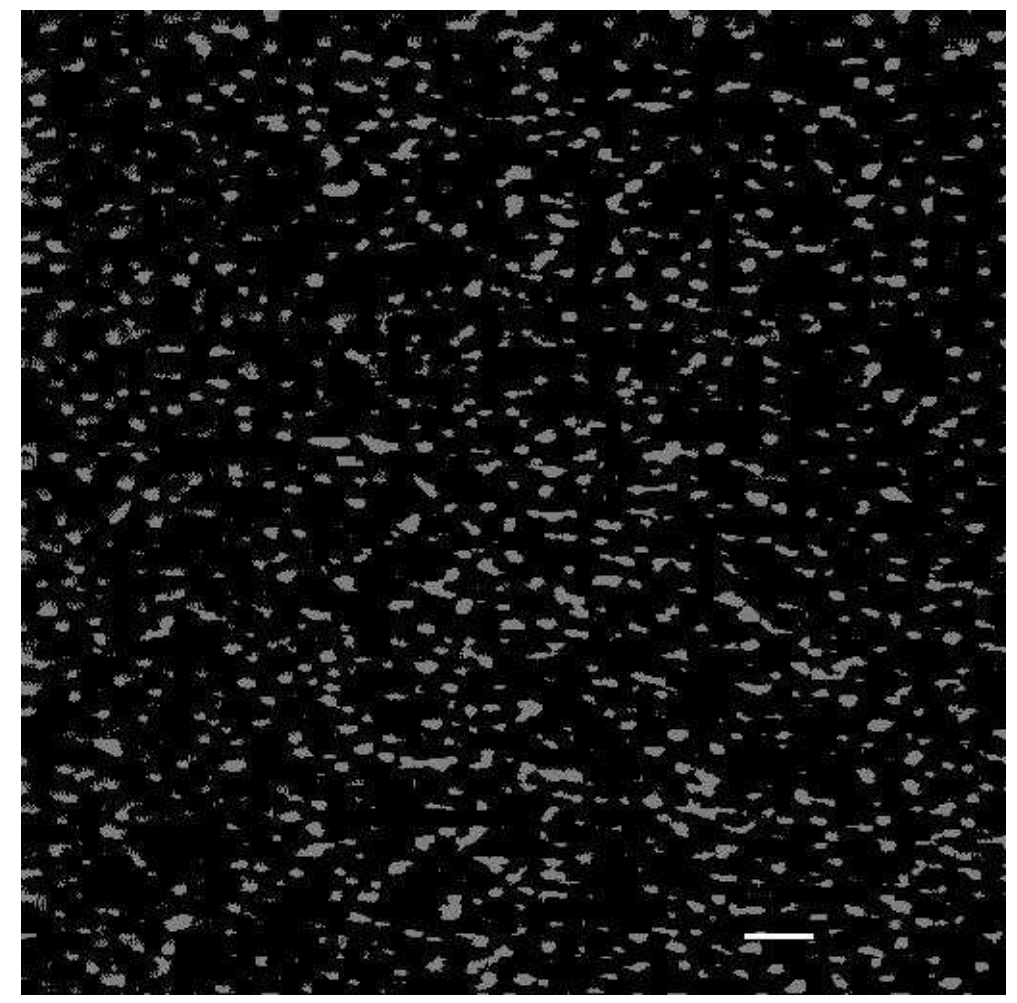

Figure 3: A sample image of a mica surface coated with fibronectin at about 1,400 molecules $/ \mu m^{2}$. The image was obtained with atomic force microscopy in aqueous medium and tentatively binarized with a height threshold of $9.2 \mathrm{~nm}$. This may resemble what is actually seen by receptors on the surface of flowing cells. Clearly, fibronectin is not organized as an assembly of isolated molecule with identical conformation. This illustrates the need of studying the molecular organization of adhesive surfaces. Bar length is $250 \mathrm{~nm}$.

When binding sites are diluted, binding event frequency decreases while the unbinding kinetics remains unchanged. Obviously, this argument may rule out the possibility that observed binding events are a combination of unimolecular and multimolecular associations. However, this is fully convincing only if it is shown than minimal detectable events are single molecular bonds.

Conclusion. While it seems reasonable to conclude that many authors actually monitored individual bond formation and dissociation, much caution is needed to prove that this happened in a given experimental situation.

\subsection{Is it possible to quantify the force dependence of unbinding rates with a flow chamber?}

Flow chambers were repeatedly used to determine the mechanical properties of single bonds 22,29 , [7, 36. The basic principle consisted of determining bond dissociation rates for different values of the wall shear stress. The force applied on the bond was derived from the force on the cell with 
simple geometrical arguments 22, 10. This procedure would be fully warranted if bond formation was always a monophasic process. However, when bond formation is multiphasic, a decrease of bond lifetime as a consequence of an increase of the wall shear rate may be ascribed to either a decrease of bond lifetime in presence of a disruptive force, or a decrease of bond strengthening due to decrease of the time allowed for bond formation before being subjected to hydrodynamic drag. As was mentioned above, unperturbed intermolecular contact may last a millisecond or less in a flow chamber, and there is much evidence to support the view that formation of a fully stable bond may require a notably higher amount of time [10, 11, 13]. It is therefore conceivable that the use of flow chambers with too high shear rates might result in the observation of multiple incompletely strengthened bonds, thus accounting for possible discrepancies with force parameters obtained with other methods.

\subsection{What is the meaning of binding frequencies determined with flow chambers?}

A notable advantage of operating flow chambers under fairly low shear rate is that cell or particle trajectories may be easily followed independently of the occurrence of any binding event. Thus, it is easy to determine the binding probability per unit length of trajectory. Now, there remains to assess the significance of this parameter. Two different hypotheses may be considered.

i) The rate limiting step might be the molecular encounter between receptors and ligands. If each encounter is conducive to bond formation, binding frequency per unit length should be independent of particle velocity.

ii) Alternatively, if the intrinsic rate of bond formation is low, contact time might be the rate limiting step. Increasing particle velocity would then be expected to decrease binding frequency.

Interestingly, recent experimental evidence suggests that both possibilities may occur 61. Indeed, when human lymphocytic Jurkat cells were made to bind to ICAM-1, a ligand of cell surface integrin LFA-1, binding frequency was inversely correlated to the wall shear rate. However, when the flow chamber surface was coated with anti-LFA-1 antibodies, binding frequency per unit length became independent of the wall shear rate. This difference is consistent with the notoriously high association rate of antibodies.

Conclusion. Before trying to extract information on molecular association rates from binding frequency measurements, it is important to check that adequate experimental conditions (i.e. ligand density and wall shear rates) have been selected.

\subsection{Is molecular on-rate an intrinsic parameter ?}

A rather subtle cause of misinterpretation consists of trying to determine a parameter that does not actually exist. A prominent example is the study of bond rupture strength with atomic force microscopy. Initial results were interpreted with the underlying assumption that there exists an intrinsic bond strength, which is true only when temperature is low enough. Similarly, $k_{\text {on }}$ determinations rely on the assumption that the probability that a ligand and a receptor form a bond during an encounter of vanishingly short duration $d t$ is proportional to $d t$. In fact, this procedure raises some difficulties. First, defining a ligand as bound to a receptor requires the determination of an arbitrary threshold, be it a minimal binding energy or capacity to resist a disruptive force during a sufficient period of time to be detected. Second, several barriers may have to be crossed for such a threshold to be reached. In this case, the binding probability may be proportional to a power of 
encounter duration that might be higher than one. An other way of describing this problem would be to recall that a rigorous study of association rate would consist of $i$ ) defining binding states, and ii) determining the probability $k_{o n}(d, t)$ that a ligand and a receptor molecules remained "bound" after remaining separated by distance $d$ during time $t$.

\subsection{The limits of the flow chamber method are set by the requirement to discriminate between actual and artefactual binding events}

First, it must be emphasized that the limits of bond analysis may be set by qualitatively different parameters when cells or microspheres are used. Indeed, an essential feature of biological cells is that they are studded with protrusions of varying size, and they may resemble ellipsoids rather than perfect spheres. This results in two difficulties : first, the region of contact between a cell and the chamber floor under flow is not accurately known. Thus, if a cell is rotating around a bound contact, a bond may remain undetectable since the cell may seem to move continuously. Conversely, a cell may seem to undergo a brief arrest if it rotates on the tip of a large protrusion 20. Thus, it was felt that it was better to use microspheres rather than cell to achieve optimal resolution in studying molecular interactions [10, 29]. Indeed, The accurate position of sphere-to-surface contact may be determined with high accuracy by calculating the position of the centroid of the sphere image. It was thus estimated that $50 \mathrm{~nm}$ accuracy was easily obtained when spheres of $2.8 \mu \mathrm{m}$ diameter were monitored. Now, three different artifactual binding events might occur. These will be considered sequentially. For this purpose, the simplest procedure consists of defining a sphere as arrested when it moved by a distance lower than some threshold $\chi$ during a given period of time $\tau$

Consequences of measurement errors. Clearly as shown on Figure 4, an error of position determination may result in artefactual short arrest of about 1 step duration. This is fairly easy to detect on visual examination of the trajectory, and this error might be detected by using a more refined way of defining arrest, such as a translation of the regression line determined on a series of steps 1. However, we did not find it warranted since it was only a minor problem as compared to other artifacts. Note that it is essential to rule out more important problems due to collisions between flowing and arrested particles. This is easily controlled by combining position and area determination, and deleting events associated with transient area increase 51]

Statistical errors due to Brownian motion. In a flow chamber, Brownian motion will result in displacements parallel to the flow (i.e. horizontal) and perpendicular to the wall (i.e. vertical). The latter displacement will generate a velocity variation proportional to the wall shear rate $G$, since the average velocity of a sphere is dependent on the distance to the wall[18. The resulting variance of the displacement $\Delta x$ during time interval $\Delta t$ is thus expected to follow the equation :

$$
<\Delta x>^{2}=a G^{2}<\Delta t>^{2}+b \Delta t
$$

An important consequence is that the hydrodynamic displacement, which is proportional to $\langle\Delta t>$ will decrease more rapidly than the Brownian displacement when the time period vanishes. This means that it is useless to increase the sampling rate for position determination. In other words, the time resolution limit for arrest detection is set by Brownian motion. Note that the validity of Equation 10 was checked both experimentally, by studying the motion of non adhesive particles, and with computer simulation. Now, for a given wall shear rate, the threshold parameters for arrest detection must be chosen in order to maximize detection sensitivity provided the proportion

\footnotetext{
${ }^{1} \mathrm{~PB}$ is indebted to Dr. D. Bensimon for pointing out the potential interest of refining arrest detection
} 


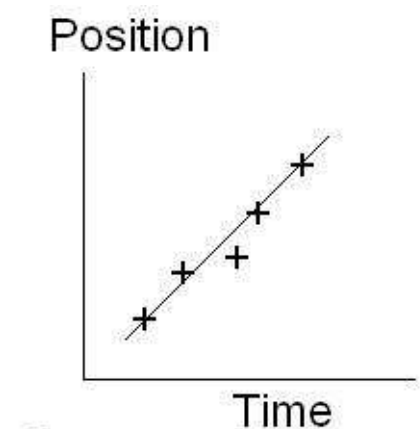

A

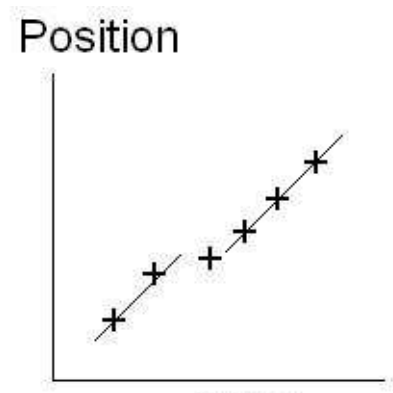

Time

Figure 4: Errors in position determination. Artefactual arrests due to errors in position determination are easily detected on visual inspection (A) and they appear as isolated points. Actual decrease of particle velocity result in translation of the regression line built on a sequenc of steps (B).

of statistical binding events remain negligible as compared to physical binding events. This means that arrest detection may be more sensitive when efficient binding molecular models are studied. On the contrary, when physical binding is unfrequent and many positions must be studied, higher threshold time $\tau$ must be selected. Thus, if 100,000 displacement steps must be scanned to detect about 100 binding events, statistical artefact probability must be lower than $10^{-4}$ in order that 90 $\%$ of detected arrests represent bona fide binding events. A possible way of reducing the importance of statistical arrests might seem to consist of increasing the wall shear rate. However, this will often reduce binding frequency. This means that the optimal conditions for studying a given molecular model must be determined somewhat empirically. A parameter that might seem worth considering is particle size. However, reducing particle size in order to improve the ratio between sphere hydrodynamic velocity and force on a bond will increase vertical brownian motion, thus decreasing the sphere residence time within binding distance of ligands. Binding efficiency might thus be drastically decreased.

\subsection{Treatment of nonspecific interactions}

A problem that is always encountered when starting to study a new molecular model is the following : how can we prove that monitored binding events are bona fide interactions between ligand and receptor binding sites?

An apparently reasonable check might consist of verifying that particles no longer bind the chamber floor when either particle or chamber coating is omitted. However, this is not a rigorous proof. Thus, that bare spheres do not bind to a ligand-coated surfaces is not a proof that receptorcoated spheres will form specific interactions with the same surface. Indeed, receptor molecules might form nonspecific interactions with the chamber surface.

Another argument might consist of checking that binding events are inhibited when the flow medium is altered in order to prevent specific ligand-receptor bonds. A frequent way of achieving this results might consist of adding divalent cation chelators since these cations are required 
for binding mediated by important adhesion receptors such as selectins, integrins or cadherins. Unfortunately, performing this test cannot be considered as a satisfactory check. Thus, in our laboratory, cadherin coated spheres displayed calcium-dependent binding to cadherin-coated surfaces, as expected. However, calcium chelation also prevented association between cadherin-coated beads and bare mica. In other words, nonspecific interactions may display a similar sensitivity to physico-chemical conditions as specific associations do.

Also, according to our experience, nonspecific interactions do not exhibit clearcut enough properties such as hight sensitivity ot rupture forces, to allow safe discrimination between the properties of specific and nonspecific bonds.

Thus, according to our experience, the safest way of proving that specific ligand-receptor bonds are indeed studied may consist of specifically inhibiting binding events with small molecular weight molecules or comparing wild-type receptors to genetically engineered molecules wiht a critical mutation located to the binding site. Thus, streptativin-biotin interactions were efficiently inhibited by adding low molecular weight biotin to the surrounding medium[11. Also, homophilic cadherin interactions were inhibited by mutating of a critical tryptophan residue into alanin [36.

\subsection{Intrinsic ambiguity of data interpretation}

A significant difficulty arose as the analysis of single molecular bonds was more and more refined. Several different models can account for a given set of experimental data. This intrinsic ambiguity was indeed quoted in several experimental $[99$ or theoretical [62 studies. The analysis of unbinding curves obtained with flow chambers clearly illustrates this point : a typical curve such as shown on Fig. 2 appears with upwards concavity that might be due to i) formation of additional bonds after initial arrest 29, ii) time-dependent strengthening of a single bond 10 or iii) existence of two different bond species with different rupture frequencies. It is possible to discriminate between (i) and (ii) by performing different experiments with varying ligand or receptor surface concentrations. However, (ii) and (iii) may be difficult to distinguish if it is accepted that a given ligand-receptor pair may form two different kinds of bonds, as was recently demonstrated 63. Indeed, when ligandreceptor interaction consists of forming a transient state with dissociation rate $k_{o f f}$ and rate of transition to a stable state $k_{t}$, the equation of the unbinding curve is readily shown to be :

$$
N(t)=\frac{N_{0}\left[k_{m} \exp \left(-\left(k_{o f f}+k_{t}\right) t\right)+k_{t}\right]}{k_{o f f}+k_{t}}
$$

Howevever, the unbinding rate of particles that may be bound either with a transient bond with dissociation rate $k_{\text {off }}$ or a stable bond is given by :

$$
N(t)=A \exp \left(-k_{o f f} t\right)+B
$$

Clearly, Equations 11 and 12 are formally identical.

Thus, more and more complementary experiments will be needed to achieve safe interpretation of experimental data.

\subsection{Accuracy of rate constant determination.}

An important point to achieve meaningful comparizon of results obtained with different techniques is te determine the accuracy of rate constant determination. A prominent cause of variation is due to counting errors. This may be illustrated by considering off-rate determinations. Computer 
simulations were performed to determine the minimum number of arrests used to build unbinding curves 30 . It was concluded that the coefficient of variation of the off-rate determination was respectively $39 \%, 25 \%, 17 \%$ and $8.5 \%$ when $25,50,100$ or 200 arrest durations were used.

Another simple estimate was obtained as follows 11 : assuming Poisson distribution for the numbers $\mathrm{A}$ and $\mathrm{B}$ of particles detached at time $\mathrm{t}$ within interval $\left[t_{1}, t_{2}\right]$ and $\left[t_{2}, \infty\right]$ after arrest, the average detachment rate during interval $\left[t_{1}, t_{2}\right]$ is $\frac{\ln (B /(A+B))}{t_{2}-t_{1}}$. The standard deviation for dissociation rate is therefore equal to $\sqrt{\left[\frac{A}{B(A+B)}\right]} /\left(t_{2}-t_{1}\right)$

\section{Conclusion.}

The aim of this brief review was essentially to summarize the new kind of information that was made available by using a flow chamber operated under low shear rate to dissect the interaction between surface-attached ligand and receptor molecules at the single bond level. The following conclusions were obtained. i) The rates of bond formation and dissociation between soluble ligand and receptors do not encompass sufficient information to account for the whole functional activity of these molecules when they are bound to cell surfaces. Indeed, parameters such as molecular length, attachment flexibility, topographical distribution on the cell surface and molecular environment strongly influence adhesive interactions. ii) The flow chamber was found to allow efficient analysis of the functions of most adhesion molecules such as selectins [25, 22, integrins [30, 44, 61, members of the immunoglobulin superfamily 29] or cadherins [36]. iii) Results obtained with this and other techniques suggest that the rupture of newly formed attachments may often be adequately described with a set of three parameters, i.e. rupture frequency and force coefficient of initial binding state and strengthening rate. More work is required to find an adequate set of parameters to describe bond formation. iv) In view of presently available evidence, it is certainly warranted to undergo systematic comparison between bond parameters obtained with different methods, including flow chambers, atomic force microscopy and biomembrane force probes, and then to determine whether these parameters give a satisfactory account of receptor function under physiological conditions. v) Another important prospect is certainly to pursue comparisons between experimental parameters and computer simulation of bond formation and dissociation 64. This is certainly a promising way of gaining more profound understanding of protein structure and function, as well as predicting functional properties of receptors of known structure. vi) However, it must be kept in mind that data yielded by flow chambers are often difficult to understand completely, and full interpretation often requires a number of controls that must be specifically to each system under study.

\section{Acknowledgments}

Part of presented work was supported by the ARC. 


\section{5 references}

\section{References}

[1] T. E. Creighton Proteins - Structure and Molecular Properties, 1st edn. (Freeman, New York, 1983).

[2] A. N. Barclay Disc. Faraday Soc.111, 345, 1998.

[3] C. M. Isacke and M. A. Horton The Adhesion Molecule FactsBook, 2nd edn. (Academic Press, London, 2000).

[4] A. F. Williams, Nature 352, 473 (1991).

[5] M. A. Lawrence and T. A. Springer Cell 65, 859, 1991.

[6] J. D. Ashwell Immunity 21, 603, 2004.

[7] K. D. Puri, S. Chen and T. A. Springer Nature 392, 930, 1998.

[8] C. Pujades et al. Mol. Biol. Cell 8, 2647, 1997.

[9] E. Perret et. al. PNAS 101, 16472, 2004.

[10] A. Pierres, A. M. Benoliel and P. Bongrand J. Biol. Chem. 270, 26586, 1995.

[11] A. Pierres, D. Touchard, A. M. Benoliel and P. Bongrand Biophys. J. 82, 3214, 2002.

[12] B. T. Marshall et al. Biophys. J. 88, 1458, 2005.

[13] F. Pincet and J. Husson Biophys. J.89, 4374, 2005.

[14] A. Pierres, A. M. Benoliel and P. Bongrand Cell Adhesion Commun. 5, 375, 1998.

[15] U. Seifert Phys. Rev. Lett. 84, 2750, 2000.

[16] A. Pierres, A. M. Benoliel and P. Bongrand J. Immunological Methods 196, 105, 1996.

[17] A. Atherton and G. V. R. Born J. Physiol. (Lond) 222, 447, 1972.

[18] A. J. R. Goldman, G. Cox and H. Brenner Chem. Engn. Sci. 22, 653, 1967.

[19] A. Pierres, A. M. Benoliel, C. Zhu and P. Bongrand Biophys. J. 81, 25, 2001.

[20] O. Tissot, A. Pierres, C. Foa, M. Delaage and P. Bongrand Biophys. J. 61, 204, 1992.

[21] O. Tissot, C. Foa, C. Capo, H. Brailly, M. Delaage and P. Bongrand J. Dispersion Science Technol. 12, 145, 1991.

[22] R. Alon, D. A. Hammer and T. A. Springer Nature 374, 539, 1995.

[23] D. W. Schmidtke and S. L. Diamond J. Cell Biol. 149, 719, 2000.

[24] S. P. Tha, J. Shuster and H. L. Goldsmith Biophys. J. 50, 1117, 1986. 
[25] G. Kaplanski, C. Farnarier, O. Tissot, A. Pierres, A. M. Benoliel, M. C. Alessi, S. Kaplanski and P. Bongrand Biophys. J. 69, 1922, 1993.

[26] G. I. Bell Science 200, 618, 1978.

[27] E. Evans, D. Berk and A. Leung Biophys. J. 64, 838, 1991.

[28] C. Zhu, M. Long, S. E. Chesla and P. Bongrand Ann. Biomed. Engineering. 30, 305, 2002.

[29] A. Pierres, A. M. Benoliel, P. Bongrand and P. A. van der Merwe Proc. Natl. Acad. Sci. USA 93, 15114, 1996.

[30] B. Masson-Gadais, A. Pierres, A-M Benoliel, P. Bongrand and J. C. Lissitzky J. Cell Sci. 112, 2335, 1999 .

[31] A. Pierres, O. Tissot and P. Bongrand, Analysis of the motion of cells driven along an adhesive surface by a laminar shear flow, in Studying Cell Adhesion, eds P. Bongrand, P. Claesson and A. S. G. Curtis (Springer-Verlag, Heidelberg, 1994), p. 157.

[32] A. Pierres, A. M. Benoliel and P. Bongrand, Initial steps of cell-substrate adhesion, in Cell Mechanics and Cellular Engineering, eds V. C. Mow, F. Guilak, R. Tran-Son-Tay and R. M. Hochmuth (Springer-Verlag, New York, 1994), p. 145.

[33] E. A. Evans and K. Ritchie Biophys. J. 72, 1547, 1997.

[34] P. Bongrand Rep. Progress. Phys. 62, 921, 1999.

[35] R. Alon, S. Chen, K. D. Puri, E. B. Finger and T. A. Springer J. Cell Biol. 138, 1169, 1997.

[36] E. Perret, A. M. Benoliel, P. Nassoy, A. Pierres, V. Delmas, J. P. Thiery, P. Bongrand and H. Feracci $E M B O J$ 21, 2537, 2002.

[37] D. F. Tees and H. L. Goldsmith Biophys. J. 71, 1102, 1996.

[38] D Kwong, D. F. Tees and H. L. Goldsmith Biophys. J. 71, 1115, 1996.

[39] S Chen and T. A. Springer Proc. natl. Acad. Sci. USA 98, 950, 2001.

[40] B. T. Marshall, M. Long, J. W. Piper, T. Yago, R. P. McEver and C. Zhu Nature 423, 190, 2003.

[41] M. Dembo, D. C. Torney, K. Saxman and D. Hammer Proc. Roy. Soc.B 234, 55, 1988.

[42] E. L. Florin, V. T. Moy and H. E. Gaub Science 264, 415, 1994.

[43] R. Merkel, P. Nassoy, A. Yeung, K. Ritchie and E. Evans Nature 397, 50, 1999.

[44] J. Vitte, A. M. Benoliel, P. Eymeric, P. Bongrand and A. Pierres Biophys. J. 86, 4059, 2004.

[45] X. Zhang, E. Vojcikiewicz and V. T. Moy Biophys. J. 83, 2270, 2002.

[46] W. Hanley, O. McCarty, S. Jadhao, Y. Tseng, D. Wirtz and K. Konstantopoulos J. Biol. Chem. 278, 10556, 2003. 
[47] P. Panorchan, M. S. Thompson, K. J. Davis, Y. Tseng, K. Konstantopoulos and D. Wirtz J. Cell Sci 119, 66, 2006.

[48] E. Perret, A. Leung, H. Feracci and E. Evans Proc. Natl. Acad. Sci. USA 101, 16472, 2004.

[49] E. Evans, A. Leung, V. Heinrich and C. Zhu Proc. Natl. Acad. Sci. USA 101, 11281, 2004.

[50] H. Eyring J. Chem. Phys. 3, 107, 1935.

[51] A. Pierres, A. M. Benoliel, P. Bongrand and P. A. van der Merwe FEBS Lett. 403, 239, 1997.

[52] A. Pierres, H. Feracci, V. Delmas, A. M. Benoliel, J. P. Thiery, and P. Bongrand Proc. Natl. Acad. Sci. USA 95, 9256, 1998.

[53] P. Hinterdorfer, W. Baumgartner, H. J. Gruber, K. Schilcher and H. Schindler Proc. Natl. Acad. Sci. USA 93, 3477, 1996.

[54] W. Baumgartner, P. Hinterdorfer, W. Ness, A. Raab, D. VestWeber, H. Schindler and D. Drenckhahn Proc. Natl. Acad. Sci. UsA. 97, 4005, 2000.

[55] K. D. Patel, M. U. Nollert and R. P. McEver J. Cell Biol. 131, 1893, 1995.

[56] S. Sabri, A. Pierres, A. M. Benoliel and P. Bongrand Biochem. Cell Biol. 73, 411, 1995.

[57] J. Huang, J. Chen, S. E. Chesla, T. Yaog, P. Mehta, R. P. McEver, C. Zhu and M. Long J. Biol. Chem. 279, 44915, 2004.

[58] C. Jeppesen, J. Y. Wong, T. L. Kuhl, J. N. Israelachvili, N. Mullah, S. Zalipsky and C. M. Marques Science 293, 465, 2001.

[59] C. Jun, M. Shimaoka, C. V. Carman, J. Takagi and T. A. Springer Proc. Natl. Acad. Sci. USA 98, 6830, 2001.

[60] T. V. Ratto, R. E. Rudd, K. C. Langry, R. L. Balhorn and M. W. McElfresh Langmuir, 2006.

[61] J. Vitte, A. Pierres, A. M. Benoliel and P. Bongrand J. Leukocyte Biol. 76, 594, 2004.

[62] I. Derenyi, D Bartolo and A. Ajdari Biophys. J. 86, 1263, 2004.

[63] S. Sivasankar, B. Gumbiner and D. Leckband Biophys. J. 80, 1758, 2001.

[64] M. V. Bayas, K. Schulten and D. Leckband Biophys. J. 84, 2223, 2003. 